\title{
Comparison of the clinical benefits for non- small cell lung cancer patients between different volume of pleural lavage fluid following video-assisted thoracoscopic lobectomy and systematic mediastinal lymph node dissection: study protocol for a randomized controlled trial
}

Jian Zhou ${ }^{1,2+}$, Chengwu Liu ${ }^{1,2+}$, Shulei Man², Mengyuan Lyu ${ }^{2}$, Hu Liao ${ }^{1,2}$, Nan Chen ${ }^{1,2}$, Yuhui Cheng ${ }^{2}$ and Lunxu Liu Li, $^{*}$

\begin{abstract}
Background: Pleural lavage is regularly performed before closing the chest wall in pulmonary surgeries to prevent pleural implantation of tumor cells and postoperative infection. However, scant data could be found in the literature regarding the optimal regimen for performing pleural lavage. To establish a proper volume of pleural lavage, we herein designed a protocol for a randomized controlled trial.

Methods: A total of 400 participants with non-small cell lung cancer undergoing video-assisted thoracoscopic surgery (VATS) lobectomy and systematic mediastinal lymph node dissection (MLND) will be randomly assigned to one of two groups: group A (500 mL pleural lavage fluid) and group B (3000 mL pleural lavage fluid). The primary outcomes include the levels of leukocytes, neutrophils, and inflammatory factors on the first postoperative day. The secondary outcomes include (i) the levels of leukocytes, neutrophils, and inflammatory factors on the second and third postoperative days; (ii) the incidence of postoperative fever on the first, second, and third postoperative days; (iii) the volumes of chest drainage within the first 3 operative days, the duration of drainage, and postoperative hospitalization; and (iv) the incidence of postoperative complications (incision infection, pain, atelectasis, hemorrhage, etc.) and the incidence of pleural effusion requiring thoracic puncture or drainage within 30 days after surgery. The main content of the analysis includes effectiveness and safety analysis. We will perform subgroup analyses to identify potential influence factors.
\end{abstract}

(Continued on next page)

\footnotetext{
* Correspondence: lunxu liu@aliyun.com

† Jian Zhou and Chengwu Liu contributed equally to this work.

'Department of Thoracic Surgery, West China Hospital, Sichuan University,

No. 37, Guoxue Alley, Chengdu 610041, Sichuan, China

${ }^{3}$ Western China Collaborative Innovation Center for Early Diagnosis and

Multidisciplinary Therapy of Lung Cancer, Sichuan University, No. 37, Guoxue

Alley, Chengdu 610041, Sichuan, China

Full list of author information is available at the end of the article
}

(c) The Author(s). 2020 Open Access This article is distributed under the terms of the Creative Commons Attribution 4.0 International License (http://creativecommons.org/licenses/by/4.0/), which permits unrestricted use, distribution, and reproduction in any medium, provided you give appropriate credit to the original author(s) and the source, provide a link to the Creative Commons license, and indicate if changes were made. The Creative Commons Public Domain Dedication waiver (http://creativecommons.org/publicdomain/zero/1.0/) applies to the data made available in this article, unless otherwise stated. 
(Continued from previous page)

Discussion: As far as we know, this will be the first randomized controlled trial to compare the clinical outcomes between different volumes of pleural lavage fluid following VATS and MLND. Findings from this trial will determine the appropriate amount of pleural lavage before chest wall closure.

Trial registration: This study was registered with the Chinese Clinical Trial Registry ( on 17 March 2019. ChiCTR 1900021950).

Keywords: Pleural lavage fluid, Pulmonary surgery, Non-small cell lung cancer, Thoracic drainage, Randomized controlled trial

\section{Background}

In pulmonary surgery, pleural lavage is routinely performed before closing the chest wall, to rinse off residual tumor cells and tissues and ideally prevent pleural implantation of tumor and postoperative infection [1]. Decades ago, it was known that even if there were no obvious malignant pleural effusion or pleural implants, tumor cells could be found in as much a third of postoperative pleural lavage [2]. Since then, accumulating data have indicated the presence of tumor cells in intraoperative pleural lavage as an independent prognostic factor [3-5]. Intraoperative pleural lavage cytology detected before closure could present a higher prognostic value than pleural lavage cytology detected before thoracotomy. Furthermore, it could guide the choice of adjuvant chemotherapy for lung cancer patients after surgery.

Although there is no guideline regarding how pleural lavage should be conducted [6], usually it involves irrigating the thoracic cavity with $0.9 \%$ sodium chloride injection varying from 20 to $2000 \mathrm{~mL}[2,7-9]$ at $38 \sim 40{ }^{\circ} \mathrm{C}$ [10]. However, there are no determinant criteria on the volume of pleural lavage fluid. If the volume of pleural lavage is too small, the residual tumor cells and tissue cannot be washed away, which may result in increased absorption of inflammatory mediators, fevers, and even severe inflammatory reactions [11]. It could affect prognosis and prolong hospitalization [12]. Furthermore, the residual tumor cells may increase the risk of recurrence [13] and metastasis [14-16]. If the volume of pleural lavage is excessive, it will cause waste of resources and prolongation of operation time. Kaneda et al. [9] found that doses of over $500 \mathrm{~mL}$ could cause false-negative results of pleural lavage cytology. Considering clinical practice and the literature $[7,8]$, we decided to test two volumes of pleural lavage: 500 and $3000 \mathrm{~mL}$.

We will prospectively enroll non-small cell lung cancer (NSCLC) patients undergoing video-assisted thoracoscopic surgery (VATS) for lobectomy and systematic mediastinal lymph node dissection (MLND). After enrollment, we will randomly allocate patients to one of two groups: group A (500 mL pleural lavage fluid) or group B (3000 mL pleural lavage fluid). Blood samples will be collected to test for leukocytes, neutrophils, and inflammatory factors. Postoperative complications, the volume of pleural drainage, and length of hospital stay will also be recorded. We aim to compare the clinical benefits for patients with NSCLC between different volumes of pleural lavage fluid following VATS lobectomy and MLND.

\section{Methods/Design}

Trial design

This is a single-blind, single-center, randomized controlled trial (Fig. 1). This study protocol adheres to the Standard Protocol Items: Recommendation for Interventional Trials (SPIRIT) statement. The SPIRIT figure (Fig. 2) summarizes the items of enrollment, intervention, and follow-up. The detailed SPIRIT checklist is also provided (Additional file 1).

\section{Study objective}

This study aims to identify the effects of different volumes of pleural lavage fluid on perioperative outcomes of patients with NSCLC following VATS lobectomy and MLND.

\section{Study location}

This study will be conducted in NSCLC patients undergoing VATS lobectomy and MLND in the Department of Thoracic Surgery, West China Hospital, Sichuan University.

\section{Recruitment \\ Recruitment of participants}

Patients eligible for this trial must comply with all the inclusion criteria and must not meet any exclusion criteria. To achieve adequate enrollment, all surgeons in the thoracic department of the hospital are informed of this trial. Each included patient will sign an informed consent form. The consent form includes (i) the detailed explanation of the study design, including backgrounds and aims of this trial; (ii) the benefits and risks of participating; and (iii) the strategy and compensation for the participants if they experience any harm as a result of trial participation. 


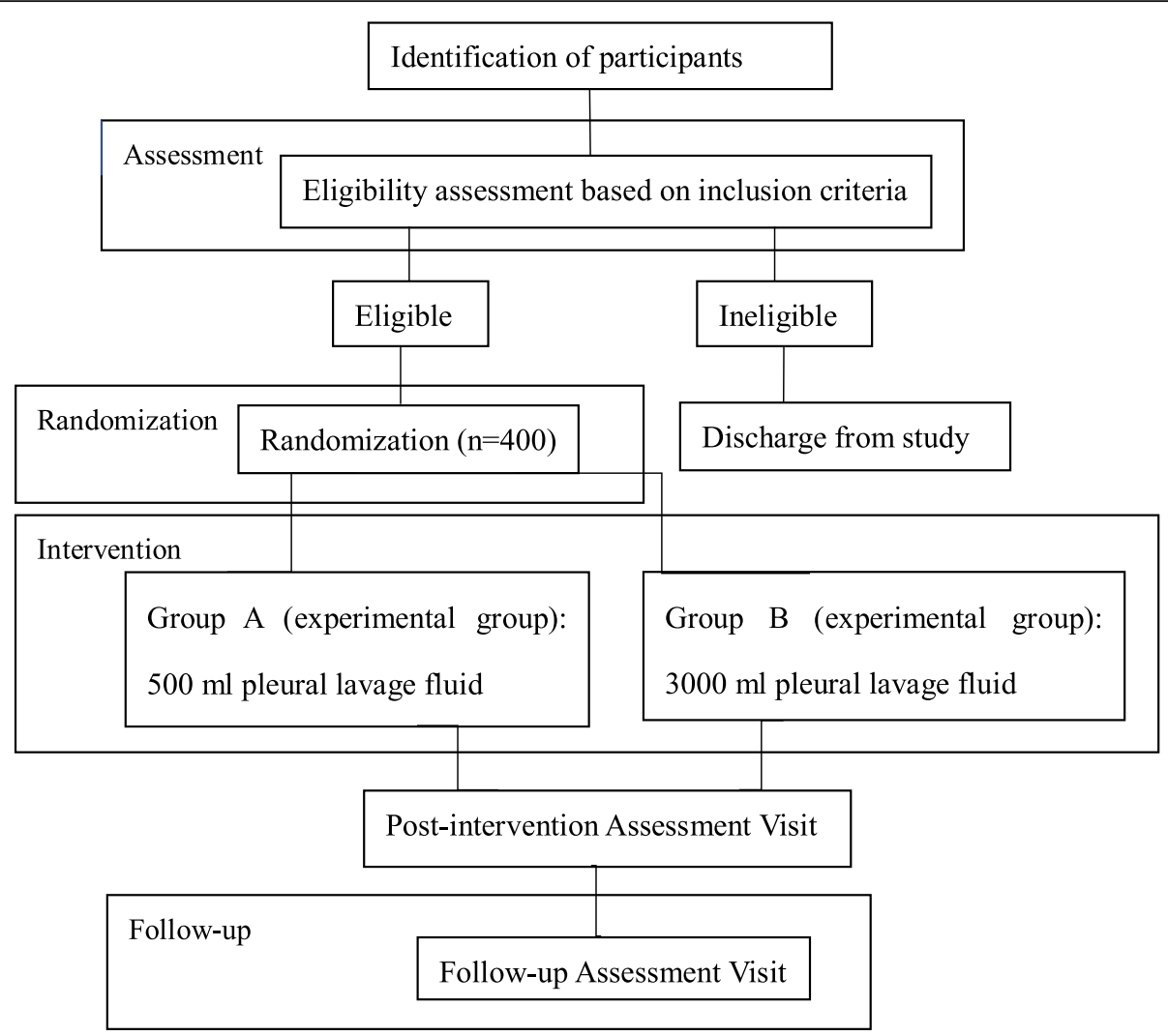

Fig. 1 Standard Protocol Items: Recommendation for Interventional Trials (SPIRIT) figure

\section{Inclusion criteria}

Patients who meet all the following criteria at the start of the treatment are eligible for this study: (i) patients between 18 and 75 years of age, (ii) patients undergoing planned VATS lobectomy and MLND, (iii) be American Society of Anesthesiologists grade I or II, (iv) essential materials such as clinical staging of lung cancer and medication were complete, (v) confirmed diagnosis of NSCLC through pathological examination after surgery, and (vi) willing to participate after reading and signing an informed consent form.

\section{Exclusion criteria}

Patients who meet any of the following criteria at the start of treatment are excluded from this study: (i) last smoked fewer than 2 weeks prior to surgery for current smokers, (ii) preoperative hydrothorax of patients was predominant, (iii) patients were pregnant or breastfeeding (females from 18 to 55 years of age should receive a pregnancy test), (iv) patients with preoperative severe mental illness, (v) patients with preoperative gastrointestinal or blood system disease, (vi) patients underwent cardiac ischemia, (vii) patients receiving preoperative radiotherapy or neoadjuvant chemotherapy, (viii) intraoperative accidents such as hemorrhage $(>500 \mathrm{~mL})$, conversion to open surgery, or cardiac arrest happened to the patients, and (ix) patients with severe postoperative bleeding or persistent air leakage, which require reoperations.

\section{Randomization and blinding}

Randomization of trial participants will be based on computer-generated random numbers prior to surgery. The random numbers will be printed and placed in consecutively numbered and separate sealed opaque envelopes, which will be opened only when a patient is enrolled and meets all inclusion criteria. The principal doctor (CL) will assign the participant to a group on the basis of the number. The research assistant should receive the notification in a timely fashion and assign patients to their study group strictly as required. This study will be single-blind. The participants will be blinded to the allocation of the participants, whereas the investigators and project manager will be unblinded. If an unexpected emergency occurs, allocation will be disclosed to the investigators, the participant will be withdrawn from this study, and a detailed explanation will be recorded if unblinding happens.

\section{Sample size}

This is the first study that focuses on the effect of different volume of pleural lavage on the clinical outcomes 


\begin{tabular}{|c|c|c|c|c|c|c|}
\hline \multirow[b]{3}{*}{ TIMEPOINT } & \multicolumn{6}{|c|}{ STUDY PERIOD } \\
\hline & \multirow{2}{*}{$\begin{array}{c}\text { Enrollment } \\
\text { 3-7days } \\
\text { before } \\
\text { surgery }\end{array}$} & \multirow{2}{*}{$\begin{array}{c}\text { Allocation } \\
\text { surgery }\end{array}$} & \multicolumn{4}{|c|}{ Follow up (postoperative) } \\
\hline & & & 1 day & 2 days & 3 days & 30 days \\
\hline \multirow{5}{*}{$\begin{array}{l}\text { ENOROLMENT: } \\
\text { Eligibility screen } \\
\text { Medical history } \\
\text { Obtaining informed } \\
\text { consent } \\
\text { Allocation }\end{array}$} & & & & & & \\
\hline & $x$ & & & & & \\
\hline & $x$ & & & & & \\
\hline & $x$ & & & & & \\
\hline & & $x$ & & & & \\
\hline \multicolumn{7}{|l|}{ INTERVENTIONS: } \\
\hline \multirow{2}{*}{$\begin{array}{l}500 \mathrm{ml} \text { pleural lavage } \\
\text { fluid }\end{array}$} & & $x$ & & & & \\
\hline & & & & & & \\
\hline \multirow{2}{*}{$\begin{array}{l}3000 \mathrm{ml} \text { pleural lavage } \\
\text { fluid }\end{array}$} & & $x$ & & & & \\
\hline & & & & & & \\
\hline \multicolumn{7}{|l|}{ ASSESSMENTS: } \\
\hline \multirow{4}{*}{$\begin{array}{l}\text { Postoperative } \\
\text { complications } \\
\text { Blood test } \\
\text { Thoracic drainage } \\
\text { Postoperative } \\
\text { hospitalization }\end{array}$} & & & & & & $x$ \\
\hline & & & & & & \\
\hline & & & & & & \\
\hline & & & & & & \\
\hline
\end{tabular}

Fig. 2 Flowchart for participants' identification, assessment, enrollment, randomization, intervention, and follow-up

following VATS lobectomy and MLND, and no reference is available to estimate the sample size. We estimated the power on the basis of the Student's $t$ test of the levels of leukocytes on the first postoperative day in each group. We estimate an effect size of 0.5 from our experience. A total of 400 participants will be recruited in this study (200 in each group). Judging from our experience, we set the dropout rate at $10 \%$ to account for inability to complete the treatment, data errors, loss to follow-up, and other unanticipated study problems. Given a type I error rate of $5 \%$, this study could provide a power of $99.72 \%$ by using G*Power (software version 3.1.3, University of Düsseldorf, Germany).

\section{Intervention}

A total of 400 NSCLC patients who are 18 to 75 years of age and who are undergoing VATS lobectomy and MLND will be recruited in our study on the basis of the inclusion and exclusion criteria. The patients will be divided into two groups:

\section{Group A (experimental group): $500 \mathrm{~mL}$ pleural lavage fluid} Before closing the chest wall, we will perform careful hemostasis and then flush the thoracic cavity with 500
$\mathrm{mL} 0.9 \%$ sodium chloride injection at $38-40{ }^{\circ} \mathrm{C}$. A $28-\mathrm{F}$ catheter will be indwelled for chest drainage.

Group B (experimental group): $3000 \mathrm{~mL}$ pleural lavage fluid We will use $3000 \mathrm{~mL} 0.9 \%$ sodium chloride injection at $38-40{ }^{\circ} \mathrm{C}$ to flush the thoracic cavity in this group. All other procedures are the same as those of group A.

\section{Study dropouts}

All recruited participants have the right to quit this study at any time for any reason based on the ethical consideration without any negative effects on their further therapy. Meanwhile, all researchers have the right to terminate the enrollment of any patients at any time within reasonable circumstances. All changes and reasons will be recorded immediately in the case report form (CRF). If the dropout rate is higher than $10 \%$, we will apply multiple imputation to avoid pitfalls involved with listwise deletion of cases. The intention-to-treat principle will be applied to analyze the data.

\section{Data management}

All data recorded in the CRF will be checked twice by two independent researchers. A data management safety 
committee composed of three independent investigators will be needed. They will supervise the study protocol adherence and participants' recruitment and confirm that the CRF is correctly completed and consistent with the original data. All data can be acquired only by the study investigators who have signed the confidential disclosure agreement. We do not plan to collect personal information about potential and enrolled participants beyond what is collected during normal hospitalization. After the trial, personally identifiable information will be omitted and placed in a separate database before any data analysis is performed. No participants' data collected in this trial will be used for other ancillary studies. The adherence to the study protocol, data collection, statistical analysis, and publication issue and related safety issues will be strictly monitored by the institutional ethics committee of West China Hospital, Sichuan University.

\section{Statistical analysis}

The main content of the analysis consists of effectiveness analysis and safety analysis. The analysis of all continuous variables will be presented as mean, standard deviation (SD), median, quartile spacing, and maximum and minimum values. The analysis of all dichotomous variables will be presented as rate, constituent ratio, and hazard ratio. We will use the $t$ test and chi-squared test, analysis of variance, and univariate and multivariate logistic regression analysis to describe our data. The factors $(P<0.15)$ in univariate analysis will be analyzed in multivariate analysis. All data will be checked twice by two independent statisticians. The two independent statisticians will also be blinded to treatment assignment. We will perform post-hoc subgroup analysis to identify potential significant factors based on age, sex, tumor location, clinical stage of tumor, resection scope, duration of surgery, the volume of intraoperative bleeding, and pathological stage of tumor. Demographics and clinical characteristics of the subjects are summarized as mean \pm SD for continuous variables and as number (percentage) for categorical variables. The difference between groups will be considered statistically significant if the $P$ value is less than 0.05 . All data will be analyzed by using SPSS (software version 25.0; SPSS Inc., Chicago, IL, USA).

\section{Study organization}

\section{Data collection and outcomes}

We will collect blood samples of patients to test leukocytes, neutrophils, and inflammatory factors. Sample collection will be performed by trained nurses. Samples will be sent to the Department of Laboratory Medicine immediately after collection. The laboratory evaluation will be conducted by technicians, who will be blinded to treatment groups. Laboratory results will be placed in an electronic chart. Specimens will be destroyed and not stored for any ancillary studies. Preoperative data will be collected within 3 days after recruitment. Surgery data will be collected within 2 days after operation. Postoperative data will be collected within 3 days after discharge. For patients discharged home, we will conduct follow-up information by phone calls and these data will be recorded within 3 days after follow-up. If there are any errors or omissions in the electronic chart, the investigator will correct them immediately. The raw data will be marked clearly when revising and will be signed by the investigator with date when the modifications are made. All data can be obtained only by the study researchers who have signed the confidential disclosure agreement.

\section{Complications}

Some postoperative complications, such as bleeding, pain at the incision site, postoperative air leak, prolonged air leak, and atelectasis, will be treated in accordance with clinical guidelines. During every ward round, conducted at least twice a day, the doctors in charge will solicit the patients' feedback and perform specific physical examinations to monitor any adverse events. All adverse events will be recorded in a timely fashion in the CRF. Postoperative follow-up will be conducted for all participants. Participants with any serious harm experienced as a result of trial participation will receive adequate compensation.

\section{Primary and secondary outcomes}

All outcomes will be defined in accordance with two previous studies $[17,18]$. The primary outcomes are the levels of leukocytes, neutrophils, and inflammatory factors-interleukin-1 $\beta$ (IL-1 $\beta$ ), IL-6, IL-8, IL-2, tumor necrosis factor-alpha (TNF- $\alpha), \mathrm{C}$-reactive protein (CRP), prostaglandin $\mathrm{E}_{2}\left(\mathrm{PGE}_{2}\right)$, and 5-hydroxytryptamine (5$\mathrm{HT}$ ) - on the first postoperative day. On the first postoperative morning, a trained nurse will collect blood samples and then send samples to test. The mean difference of the levels of leukocytes, neutrophils, and inflammatory factors will be compared between the two groups.

The secondary outcomes are (i) the levels of leukocytes, neutrophils, and inflammatory factors (IL-1 $\beta$, IL-6, IL-8, IL-2, TNF- $\alpha$, CRP, PGE $_{2}$, and 5-HT) on the second and third postoperative days; (ii) the incidence of postoperative fever on the first, second, and third postoperative days; (iii) the volumes of chest drainage within the first 3 operative days, the duration of drainage, and postoperative hospitalization; and (iv) the incidence of postoperative complications (incision infection, pain, atelectasis, hemorrhage, etc.) and the incidence of pleural effusion requiring thoracic puncture or drainage within 30 days after surgery. 


\section{Protocol amendments}

The current protocol is version 1.0 (September 25, 2018). Any amendment to the protocol that may affect the process of study or the benefits and risks to participants will require the agreement of the ethics committee.

\section{Discussion}

It is essential to flush the thoracic cavity before chest wall closure. However, scant data could be found in the literature. The most frequently used method is to flush the thoracic cavity with $0.9 \%$ sodium chloride injection heated nearly to the temperature of the human body at $38 \sim 40^{\circ} \mathrm{C}$. No determinant criteria on volume of pleural lavage fluid have been built. If the volume of pleural lavage is too small, the residual tumor cells and tissue cannot be washed away, which may result in increased absorption of inflammatory mediators, fever, and even severe inflammatory reactions and will affect prognosis and prolong hospital stay. Furthermore, the residual tumor cells may increase the risk of recurrence and metastasis. If the volume of pleural lavage is too high, it will cause waste of resources and prolongation of operation time.

The study will enroll 400 NSCLC patients undergoing VATS lobectomy and MLND and divide them into two groups. This study aims to find out whether different volumes of pleural lavage fluid $(0.9 \%$ sodium chloride injection) have different effects on prognosis of NSCLC patients measuring by some important clinical indices such as the plasma levels of leukocytes, neutrophils, inflammatory factors, and the incidence of fever after operation were observed 1 to 3 days after operation.

However, the study has some limitations. First, it is a single-center trial, which will restrict its generalizability, so a multiple-center large-sample clinical trial is warranted in the future. Second, the anesthesiologist and surgeons in charge of the intraoperative part of the study cannot be blinded to this study group regarding the safety. Third, the hospitalization time may be different across participants, which may bring effects on the prognosis of patients with NSCLC. Fourth, larger patients may need different volumes of pleural lavage.

\section{Conclusion}

This study is the first randomized controlled trial aiming to compare the clinical benefits for NSCLC patients between different volumes of pleural lavage fluid following video-assisted thoracoscopic lobectomy and systematic lymph node dissection. This study may help to develop a standardized procedure of pleural lavage before closing the thoracic cavity in patients undergoing lung cancer surgery.

\section{Trial status}

This study is not yet open for recruitment. This trial was scheduled to begin in July 2019 and to end in July 2021.

\section{Supplementary information}

Supplementary information accompanies this paper at https://doi.org/10. 1186/s13063-020-4146-1.

Additional file 1. SPIRIT (Standard Protocol Items: Recommendation for Interventional Trials) 2013 Checklist.

Additional file 2. The items from the World Health Organization Trial Registration Data Set.

\section{Abbreviations}

5-HT: 5-hydroxytryptamine; CRF: Case reported form; CRP: C-reactive protein; IL: Interleukin; MLND: Mediastinal lymph node dissection; NSCLC: Non-small cell lung cancer; PGE: Prostaglandin $\mathrm{E}_{2}$; SD: Standard deviation; SPIRIT: Standard Protocol Items: Recommendation for Interventional Trials; TNF-a: Tumor necrosis factor-alpha; VATS: Video-assisted thoracoscopic surgery

\section{Acknowledgments}

We give sincere thanks to Weelic Chong from the Sidney Kimmel School of Medicine, Thomas Jefferson University, Philadelphia, PA, USA, for his help with the English language editing of the manuscript.

\section{Authors' contributions}

$L L$, JZ, and $C L$ conceived of the study and finished its design and coordination. JZ, CL, SM, and ML developed the protocol and collected data. $\mathrm{JZ}, \mathrm{CL}, \mathrm{HL}$, and $\mathrm{LL}$ are responsible for the operations. NC and $\mathrm{YC}$ participated in statistical analysis. JZ and CL drafted the manuscript. LL financially supported this study. All authors read and approved the final manuscript.

\section{Funding}

This work was supported by the Key Science and Technology Program of Sichuan Province, China (2016FZ0118) (to LL). The study funders played no role in the study design, writing of the protocol, or the decision to submit the report for publication. The study funders will play no role and have no authority over the collection, management, analysis, or interpretation of data.

\section{Availability of data and materials}

The results of this trial will be published in an international peer-reviewed journal and presented at international scientific meetings. No later than three years after the publication of the results of this trial, we will deliver a completely deidentified data set to an appropriate data archive for sharing purposes. Any data request will be sent to the corresponding author and considered carefully.

\section{Ethics approval and consent to participate}

Ethics approval has been obtained from the institutional ethics committee for clinical research of West China Hospital, Sichuan University (2018-417). This study was registered with the Chinese Clinical Trial Registry (ChiCTR 1900021950) on 17 March 2019. The URL of the trial registry record is http://www.chictr.org.cn/ showprojen.aspx?proj=37003 (see Additional file 2). Only participants who can read and provide written informed consent will be recruited.

\section{Consent for publication}

Not applicable.

\section{Competing interests}

The authors declare that they have no competing interests.

\section{Author details}

'Department of Thoracic Surgery, West China Hospital, Sichuan University, No. 37, Guoxue Alley, Chengdu 610041, Sichuan, China. 'West China School of Medicine, Sichuan University, No. 37, Guoxue Alley, Chengdu 610041, Sichuan, China. ${ }^{3}$ Western China Collaborative Innovation Center for Early Diagnosis and Multidisciplinary Therapy of Lung Cancer, Sichuan University, No. 37, Guoxue Alley, Chengdu 610041, Sichuan, China. 
Received: 4 June 2019 Accepted: 6 February 2020

Published online: 27 February 2020

\section{References}

1. Toufektzian L, Sepsas E, Drossos V, Gkiozos I, Syrigos K. Pleural lavage cytology: where do we stand? Lung Cancer. 2014;83:14-22.

2. Enatsu S, Yoshida J, Yokose T, Nishimura M, Nishiwaki Y, Shirakusa T, et al. Pleural lavage cytology before and after lung resection in non-small cell lung cancer patients. Ann Thorac Surg. 2006;81:298-304.

3. Shoji F, Yamazaki K, Kouso H, Mori R, Takeo S. The impact of pleural lavage cytology both before and after lung resection on recurrence of non-small cell lung cancer. Ann Thorac Surg. 2016;101:2141-6.

4. Yanagawa N, Shiono S, Abiko M, Abe M, Watanabe K, Watanabe I, et al. Positive intraoperative pleural lavage cytology is a predictive marker of disease recurrence in stage I lung adenocarcinoma. Interact Cardiovasc Thorac Surg. 2014;18:621-5.

5. Nakamura T, Otsuki Y, Nakamura H, Funai K. Pleural lavage cytology afte lung resection in patients with non-small cell lung cancer and the feasibility of $20 \mathrm{~mL}$ saline solution. Asian J Surg. 2019;42:283-9.

6. Noppen M, De Waele M, Li R, Gucht KV, D'Haese J, Gerlo E, et al. Volume and cellular content of normal pleural fluid in humans examined by pleural lavage. Am J Respir Crit Care Med. 2000;162:1023-6.

7. Shintani Y, Ohta M, Iwasaki T, Ikeda N, Kanou T, Tomita E, et al. Intraoperative pleural lavage cytology after lung resection as an independent prognostic factor for staging lung cancer. J Thorac Cardiovasc Surg. 2009;137:835-9.

8. Nakao M, Hoshi R, Ishikawa Y, Matsuura Y, Uehara H, Mun M, et al. Prognosis of non-small-cell lung cancer patients with positive pleural lavage cytology. Interact Cardiovasc Thorac Surg. 2015;20:777-82.

9. Kaneda M, Yokoi K, Ito S, Niwa H, Takao M, Kondo R, et al. The value of pleural lavage cytology examined during surgery for primary lung cancer. Eur J Cardiothorac Surg. 2012:41:1335-41.
10. Hooper CE, Edey AJ, Wallis A, Clive AO, Morley A, White P, et al. Pleural irrigation trial (PIT): a randomised controlled trial of pleural irrigation with normal saline versus standard care in patients with pleural infection. Eur Respir J. 2015:46:456-63.

11. Ts'Ai YC, Keng CC, Fan YS. Intrapleural irrigation for reducing postresectional fever and pleural reaction. Chin Med J. 1959;78:313-21.

12. Higashiyama M, Doi O, Kodama K, Yokouchi H, Tateishi R, Horai T, et al. Pleural lavage cytology immediately after thoracotomy and before closure of the thoracic cavity for lung cancer without pleural effusion and dissemination: clinicopathologic and prognostic analysis. Ann Surg Oncol. 1997:4:409-15

13. Satoh $Y$, Hoshi R, Ishikawa $Y$, Horai T, Okumura S, Nakagawa K. Recurrence patterns in patients with early stage non-small cell lung cancers undergoing positive pleural lavage cytology. Ann Thorac Surg. 2007;83:197-202.

14. Kameyama K, Okumura N, Miyaoka E, Asamura H, Yoshino I, Tada H, et al. Prognostic value of intraoperative pleural lavage cytology for non-small cell lung cancer: the influence of positive pleural lavage cytology results on T classification. J Thorac Cardiovasc Surg. 2014;148:2659-64.

15. Mazza F, Ferrari E, Maineri P, Dozin B, Ratto GB. Pleural lavage cytology predicts recurrence and survival, even in early non-small cell lung cancer. Surg Today. 2015;45:322-8.

16. Hanagiri T, Takenaka M, Oka S, Baba T, Shigematsu Y, Nagata Y, et al. Pleural lavage cytology immediately after thoracotomy in patients with completely resected non-small cell lung cancer. Int Surg. 2011;96:171-5.

17. Saldanha IJ, Dickersin K, Wang X, Li T. Outcomes in Cochrane systematic reviews addressing four common eye conditions: an evaluation of completeness and comparability. PLoS One. 2014;9:e109400.

18. Zarin DA, Tse T, Williams RJ, Califf RM, Ide NC. The ClinicalTrials.gov results database--update and key issues. N Engl J Med. 2011;364:852-60.

\section{Publisher's Note}

Springer Nature remains neutral with regard to jurisdictional claims in published maps and institutional affiliations.
Ready to submit your research? Choose BMC and benefit from:

- fast, convenient online submission

- thorough peer review by experienced researchers in your field

- rapid publication on acceptance

- support for research data, including large and complex data types

- gold Open Access which fosters wider collaboration and increased citations

- maximum visibility for your research: over $100 \mathrm{M}$ website views per year

At BMC, research is always in progress.

Learn more biomedcentral.com/submissions 